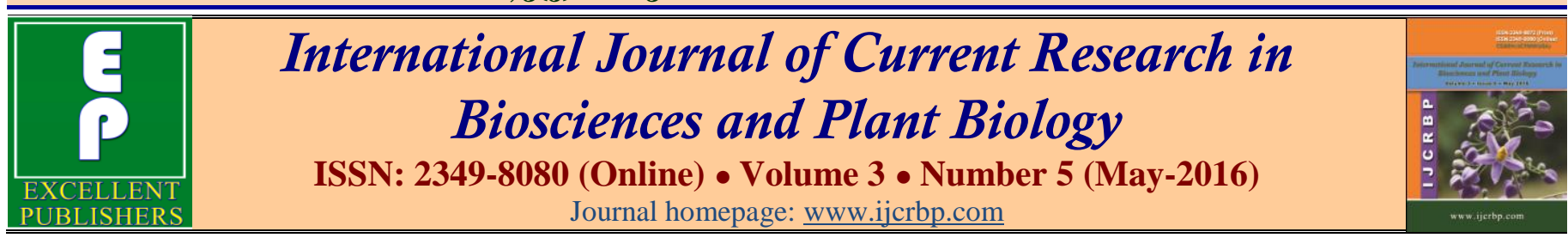

\title{
Assessment of Blood Pressure Control in Sudanese Patients with Type 2 Diabetes Mellitus
}

\author{
Abdel Salam Mohamed Hamad Elfaki ${ }^{*}$ and Mohamed Ahmed Agab² \\ $1 \& 2$ MD Medicine, Faculty of Medicine and Health Sciences, University of Kordofan, Sudan \\ *Corresponding author.
}

\begin{abstract}
A b stract
The coexistence of hypertension and diabetes mellitus increases the incidence of cardiovascular events and long-term morbidity and mortality. The aim of this study was the assessment of blood pressure control in Sudanese patients with type 2 diabetes mellitus. This descriptive, cross-sectional study includes 124 type 2 diabetic patients who were seen at the health insurance outpatient clinic in Elobied city. A questionnaire was designed for collection of socioeconomic data (gender, age, residence, occupation, and level of education) and duration of diabetes mellitus, duration of hypertension, treatment of diabetes mellitus, and treatment of hypertension. Hypertension was defined as blood pressure $\geq 140 / 90$ or the use of antihypertensive medications. Blood pressure was measured twice in sitting position in the same visit for all patients. Fifty patients were males (40\%), while $60 \%$ were females. More than half (53.2\%) of the patients belong to age group 41 - 60 years. Nearly half $(46 \%)$ of the patients were housewives. Fifty eight $(46.8 \%)$, patients were illiterate. Seventy (56.5\%), patients were hypertensive, while $54(43.5 \%)$, patients were non-hypertensive. The antihypertensive drugs which were used include: calcium channel blockers, angiotensin receptor blockers, and angiotensinconverting enzyme inhibitors, which were used by $41 \%, 38 \%$ and $14 \%$ of patients respectively. Sixteen (23\%), patients used combination of antihypertensive drugs in form of amlodipine and angiotensin receptor blockers or angiotensin-converting enzyme inhibitors. The mean systolic blood pressure in hypertensive patients was $150 \pm 23 \mathrm{mmHg}$ and the mean diastolic blood pressure was $86 \pm 13 \mathrm{mmHg}$. Most patients with diabetes require combination therapy to attain a blood pressure of less than 130/80 $\mathrm{mmHg}$. The choice of drugs should always include a ACE inhibitor or angiotensin receptor blocker and should usually include a diuretic. If additional therapy is needed a calcium channel blocker, beta blocker or alpha blocker may be used.
\end{abstract}

\section{Article Info}

Accepted: 13 April 2016

Available Online: 06 May 2016

Ke y w o r d s

Angiotensin receptor blockers Angiotensin-converting enzyme inhibitors

Calcium channel blockers

Diabetes mellitus

\section{Introduction}

Hypertension is a common comorbidity in patients with diabetes, and adequate control of blood pressure significantly reduces the risk of macrovascular and microvascular complications. The use of angiotensinconverting enzyme inhibitors may slow progression to kidney failure and cardiovascular mortality; these agents are preferred therapy for managing coexisting hypertension and diabetes. Angiotensin receptor blockers 
(ARBs) can prevent progression of diabetic kidney disease and are a first -line alternative for patients intolerant of angiotensin-converting enzyme (ACE) inhibitors.

Thiazide diuretics provide additional antihypertensive effects when combined with ACE inhibitors or ARBs. With lower doses of these drugs, the risk of clinically significant metabolic alteration is minimal. Beta blockers and calcium channel blockers also have beneficial effects in managing hypertension in patients with diabetes. Beta blockers reduce cardiovascular events and are useful in a multidrug regimen. Dihydropyridine calcium channel blockers should be reserved for patients intolerant of preferred agents or those who need additional therapy to achieve target blood pressure. Many patients with diabetes require combination therapy with multiple antihypertensive agents (Whalen, 2008).

Blood pressure should be controlled with the most appropriate drugs as well as tight blood glucose control in patients with diabetes mellitus. The blood pressure goal in diabetic patients should be below 140/90 $\mathrm{mmHg}$, probably down to $130-135 / 85 \mathrm{mmHg}$. To reach this blood pressure goal, intensive lifestyle intervention and often combination of different antihypertensive drugs must be initiated. In combination treatment, a blocker of the rennin-angiotensin system should be included, and according to the results of ACCOMOLISH trial, a combination of a rennin-angiotensin system blocker and calcium channel blocker should probably be the first choice (Aksnes et al, 2012).

The health insurance centers are well established clinics which provide health services to a large sector of population including diabetic patients. This study was conducted to assess blood pressure control in Sudanese patients with type 2 diabetes mellitus.

\section{Patients and methods}

This descriptive, cross-sectional study includes 124 type 2 diabetic patients who were seen at the health insurance outpatient clinic in Elobied city. A questionnaire was designed for collection of socioeconomic data (gender, age, residence, occupation, and level of education) and duration of diabetes mellitus, duration of hypertension, treatment of diabetes mellitus, and treatment of hypertension. Hypertension was defined as blood pressure $\geq 140 / 90$ or the use of antihypertensive medications. Blood pressure was measured twice in sitting position in the same visit for all patients.

\section{Results}

Fifty patients were males (40\%), while $60 \%$ were females. More than half $(53.2 \%)$ of the patients belong to age group 41- 60 years. The prevalence rate of hypertension in the study population was $56.5 \%$. All male diabetic patients involved in this study were hypertensive, while the majority of the female patients were non-hypertensive (Table 1). All housewives included in this study were hypertensive, while all workers were non-hypertensive (Table 2).

Table 1. Patient's gender and status of blood pressure.

\begin{tabular}{llll}
\hline \multirow{2}{*}{ Gender } & \multicolumn{2}{l}{ Hypertension } & Total \\
\cline { 2 - 4 } & Present & Absent & \\
\hline Male & 50 & 0 & 50 \\
Female & 20 & 54 & 74 \\
Total & 70 & 54 & 124 \\
\hline
\end{tabular}

Table 2. Patient's occupation and status of blood pressure

\begin{tabular}{llll}
\hline \multirow{2}{*}{ Occupation } & \multicolumn{2}{l}{ Hypertension } & \multirow{2}{*}{ Total } \\
\cline { 2 - 3 } & Present & Absent & \\
\hline Farmer & 12 & 00 & 12 \\
Housewife & 57 & 00 & 57 \\
Employee & 01 & 23 & 24 \\
Worker & 00 & 18 & 18 \\
Merchant & 00 & 06 & 06 \\
Other & 00 & 07 & 07 \\
Total & 70 & 54 & 124 \\
\hline
\end{tabular}

More than one third (33\%), of patients used amlodipine (a calcium channel blocker), as monotherapy for blood pressure control. In this group of patients, the mean systolic blood pressure was $147 \pm 21 \mathrm{mmHg}$ and the mean diastolic blood pressure was $81 \pm 7.4 \mathrm{mmHg}$.

Patients who received a combination of calcium channel blocker (amlodipine) and angiotensin-converting enzyme inhibitor (lisinopril), had a mean systolic and diastolic blood pressure $136 \pm 13 \mathrm{mmHg}$ and $84 \pm 13 \mathrm{mmHg}$ respectively, While patients who received lisinopril as a monotherapy, had systolic and diastolic blood pressure of $148 \pm 12 \mathrm{mmHg}$ and $82 \pm 8 \mathrm{mmHg}$ respectively.

Patients who received an angiotensin receptor blocker (candesartan) as monotherapy, had systolic and diastolic blood pressure of $152 \pm 16 \mathrm{mmHg}$ and $92 \pm 12 \mathrm{mmHg}$ respectively. Common antihypertensive drugs used include: amlodipine, candesartan, losartan and lisinopril. These drugs are used both as monotherapy and in combination as shown in Table 3. Only four patients used beta blocker (bisoprolol), as part of combination therapy, while no patient used thiazide diuretic. 
Table 3. Types of drugs and status of blood pressure.

\begin{tabular}{lll}
\hline \multirow{2}{*}{ Drug $(\mathbf{s})$} & Blood pressure & \\
\cline { 2 - 3 } & Systolic $(\mathbf{m m H g})$ & Diastolic $(\mathbf{m m H g})$ \\
\hline Amlodipine + Losartan & $158 \pm 28$ & $90 \pm 35$ \\
Amlodipine & $147 \pm 21$ & $81 \pm 70$ \\
Candesartan & $152 \pm 16$ & $92 \pm 12$ \\
Amlodipine + Candesartan & $143 \pm 34$ & $80 \pm 12$ \\
Amlodipine + Lisinopril & $136 \pm 13$ & $84 \pm 13$ \\
Lisinopril & $148 \pm 12$ & $82 \pm 80$ \\
\hline
\end{tabular}

\section{Discussion}

Hypertension is a common comorbidity in patients with type 2 diabetes. In this study $56 \%$ of diabetic patients were hypertensive. This finding is similar to reports from India (Ganesh and Viswanathan, 2000).

The current experimental evidence suggests that the diastolic blood pressure goal in patients with type 2 diabetes should be $80 \mathrm{mmHg}$; ongoing studies may suggest an even lower diastolic target. While the optimal level of systolic blood pressure control has not been rigorously established, it may be reasonable to target systolic blood pressure of $135 \mathrm{mmHg}$ (Vijan and Hayward, 2003). In this study the mean systolic and diastolic blood pressure was $150 \pm 23 \mathrm{mmHg}$ and $86 \pm$ $13 \mathrm{mmHg}$ respectively, both were higher than the target blood pressure levels.

In a study done by Flak and Hamaty (1999) on treatment of hypertension in African-American diabetics, they reported that in more aggressive treatment, targets for diabetics will not be reached with most currently available single antihypertensive agents in many African - Americans, and only 50-60\% of hypertensive can be controlled with single drug therapy, a percentage that falls dramatically in persons with stage 3 hypertension and renal insufficiency, thereby necessitating the use of combination drug therapy.

In this study $33 \%$ of patients used a calcium channel blocker (amlodipine) as a monotherapy for blood pressure control and their blood pressure almost reaching the optimal level with mean systolic and diastolic blood pressure $147 \pm 21 \mathrm{mmHg}$ and $81 \pm 7.4 \mathrm{mmHg}$ respectively, yet in the presence of diabetes; an angiotensin-converting enzyme inhibitor or angiotensin receptor blocker should be added to reach the target blood pressure level beside the recognized benefits of these drugs such as the nephro-protective action (Frank, 2008).
Patients who used candesartan as monotherapy had a mean systolic blood pressure of $152 \pm 16 \mathrm{mmHg}$ and a mean diastolic blood pressure of $92 \pm 12 \mathrm{mmHg}$, which was higher than the target of blood pressure in diabetics, and the same can be said about blood pressure in patients who used losartan or lisinopril as monotherapy , so in blacks, as mentioned in many studies, the responsiveness to monotherapy with angiotensin- converting enzyme inhibitors, angiotensin receptor blockers and beta blockers may be less than the responsiveness to diuretics and calcium channel blockers. It is well recognized that these differences in responsiveness are corrected when diuretics are added. The International Society of Hypertension in Blacks guidelines realizes that most patients will require combination therapy; many of them first line, to reach appropriate blood pressure goals (Ferdinand and Armani, 2007).

No patient in this study used diuretics for the treatment of hypertension as a monotherapy or part of a combination therapy, and this may be due to the traditional fear of the metabolic effects of thiazide diuretics, although several studies reported that thiazide diuretics have shown to benefit patients with diabetes and systolic hypertension. On the other hand, low dosages of thiazides are generally well tolerated and not associated with adverse metabolic effects (Devore and Bauer, 2002). Several studies showed that treatment with indapamide reduces the level of microalbuminia in patients with type 2 diabetes (Mogensen, 2003).

In this study, only four patients used a beta blocker (bisoprolol) as part of combination therapy and this is because traditionally, the use of beta blockers in diabetes has been discouraged because of adverse metabolic effects and the masking of hypoglycemic symptoms. Beta blockers therapy can be advantageous in many patients with diabetes because of its proven ability to decrease cardiovascular morbidity and mortality in persons with atherosclerotic heart disease. Cardioselective beta blockers are preferred over the non- 
selective type because the former are associated with less blunting of hypoglycemic awareness and less elevation of lipid and glucose (Devore and Bauer, 2002).

\section{Conclusion}

Most patients with diabetes require combination therapy to attain a blood pressure of less than 130/80 $\mathrm{mmHg}$. The choice of drugs should always include an ACE inhibitor or angiotensin receptor blocker and should usually include a diuretic. If additional therapy is needed calcium channel blocker, beta blocker or alpha blocker may be used.

\section{Conflict of interest statement}

Authors declare that they have no conflict of interest.

\section{References}

Aksnes, T.A., Sharn, S.N., Kjeldsen, S.E., 2012. Treatment of hypertension in diabetes: what is the best therapeutic option? Expert Rev. Cardiovasc. Ther. 10(6), 727-734.
Devore, V.S., Bauer, D.W., 2002. Controlling hypertension in patients with diabetes. Am. Fam. Physician. 66(7), 12091215.

Ferdinand, K.C., Armani, A.M., 2007. The management of hypertension in African-Americans. Crit. Pathw. Cardiol. 6(2), 67-71.

Flak, J.M., Hamaty, M., 1999. Difficult-to-treat hypertensive population: focus on African - Americans and people with type 2 diabetes. J. Hypetens. Suppl. 17(1), 19-24.

Frank, J., 2008. Managing hypertension using combination therapy. Am. Fam. Physician. 77(9), 1279-1286.

Ganesh, J., Viswanathan, V., 2000. Management of diabetic hypertensives. Indian J. Endocr. Metab. 15(4), 374-379.

Mogensen, C.E., 2003. New treatment guidelines for a patient with diabetes and hypertension. J. Hypertens. Suppl. 21(1), 25-30.

Vijan, S., Hayward, R.A., 2003. Treatment of hypertension in type 2 diabetes mellitus: Blood pressure goals, choice of agents, and setting priorities in diabetes care. Ann Intern Med. 138(7), 593-602.

Whalen, K.C., 2008. Pharmacologic management of hypertension in patients with diabetes. Am. Fam. Physician. 78(11), 1277-1282.

\section{How to cite this article:}

Hamad Elfaki, A., Agab, M. A., 2016. Assessment of blood pressure control in Sudanese patients with type 2 diabetes mellitus. Int. J. Curr. Res. Biosci. Plant Biol. 3(5), 80-83.

doi: http://dx.doi.org/10.20546/ijcrbp.2016.305.012 